\title{
Utilizing Healthcare Developments, Demographic Data with Statistical Techniques to Estimate the Diarrhoea Prevalence in India
}

\author{
Shweta Srivastava ${ }^{* *}$, Vatsalya Vatsalya ${ }^{2 \#}$, Ashoo Arora ${ }^{3}$, Kashmiri L. Arora ${ }^{4}$, Robert Karch ${ }^{2 *}$ \\ ${ }^{1}$ School of Medicine, Georgetown University, Washington DC, USA; ${ }^{2}$ College of Arts and Sciences, American University, Wash- \\ ington DC, USA; ${ }^{3}$ Department of Pediatrics, Ashirwad Hospital and Research Center, Dehradun, India; ${ }^{4}$ Department of Veterinary \\ Science, Fort Valley State University, Fort Valley, USA. \\ Email: *Shweta200in@gmail.com; *rkarch@american.edu
}

Received November $8^{\text {th }}, 2011$; revised December $10^{\text {th }}, 2011$; accepted December $30^{\text {th }}, 2011$

\begin{abstract}
Diarrhoea is one of the leading causes of morbidity and mortality in developing countries in Africa and South Asia such as India. Prevalence of diarrheal diseases in those countries is higher than developed western world and largely has been associated with socio-economic and sanitary conditions. However, present available data has not been sufficiently evaluated to study the role of other factors like healthcare development, population density, sex and regional influence on diarrheal prevalence pattern. Study was performed to understand the relationship of diarrheal prevalence with specific measures namely; healthcare services development, demographics, population density, socio-economic conditions, sex, and regional prevalence patterns in India. Data from Annual national health reports and other epidemiological studies were included and statistically analyzed. Our results demonstrate significant correlation of the disease prevalence pattern with certain measures like healthcare centers, population growth rate, sex and region-specific morbidity. Available information on sanitation like water supply and toilet availability and socioeconomic conditions like poverty and literacy measures could only be associated as trends of significance. This study can be valuable for improvisation of appropriate strategies focused on important measures like healthcare resources, population growth and regional significances to evaluate prevalence patterns and management of the diarrhoea locally and globally.
\end{abstract}

Keywords: Demographics; Diarrhoea; Healthcare; India; Prevalence

\section{Introduction}

Diarrheal disease prevalence has become a global health concern in the last few decades. Contaminated food and water supply are the most common causes of the diarrheal diseases and can be attributed to viral, bacterial or parasitic ingestion. In 1998, diarrhoea was estimated to have killed 2.2 million people worldwide [1]. Prevalence of diarrhoea is multi factorial and relies upon many factors like climate, host factor, pathogen virulence, poor hygiene and socioeconomic conditions. Incidents in developing countries are much higher due to poor hygienic conditions, sanitation and limited access to healthcare services. In Southeast Asia and Africa, diarrhoea is responsible for as much as $8.5 \%$ and $7.7 \%$ of all deaths respectively [1]. International efforts to decrease the morbidity and mortality include World Health Organization's initiative for a Diarrheal Diseases Control Program, whose objectives are to reduce diarrheal outbreaks espe-

\footnotetext{
${ }^{*}$ Corresponding author.

"These two authors have contributed equally as first authors.
}

cially in developing countries by improving the standard of treatment [2]. Diarrheal diseases are also frequently found in western world [3]; the risk groups include travellers, recent immigrants, children and institutionalized populations. However, there is well documented surveillance data available of the public resources in the western world to combat and manage the disease [4-9]. On the other hand, there are only few surveillance data available for the developing countries and have not been sufficiently interpreted for the relationships between the causes, development of prevalence and factors associated with proliferation [10-15]. Evaluation of the incidents and the associated measures that influence the disease outbreaks, progressions and demographic population has therefore become very significant to characterize and understand the associations to develop better management and planning to counteract with this condition. India, having high incident rate of diarrhoea previously; and being forefront among fast-growing nations to provide adequate health information support; matches into a desirable model to 
conduct analytical evaluation. Study is conducted to identify the relationships of measures with the prevalence; mapping of locations and characterization of the measures that may demonstrate co-occurring patterns with diarrheal incidents annually thereby elucidating present and impending disease paradigm in India. Methodology is structured towards collection of the diarrheal incidents and the measures from reliable sources; and to analyse their relationship, statistically as well as comparing with relevant publications. Evaluation of the measures has been conducted from the available state-wide national healthcare data in India comparing socioeconomic, healthcare, literacy, sanitation, poverty and demographic measures with the course of diarrheal prevalence. This study is aimed to supplement the understanding of the paradigm between the disease and the relevant measures, which can help improvise health-monitoring system and strengthen preventive strategies and management of the disease efficiently; and to develop appropriate programs; utilize and direct available resources efficiently for future disease management effectively. The outcome evaluations would also provide propositions to use similar methodologies at other diarrhoea prone locations for disease evaluation and strategy developments.

\section{Data and Methodology}

The methodology is divided into collection of data, their analysis and development of relationship combining with the statistical outcomes and information based on relevant studies.

\subsection{Data Collection}

Data source for the study has been collected from publications issued by state and central governmental agencies in India. We intended to collect information of population study relevant to demographical, healthcare and community system, availability of clean drinking water and literacy. Majority of the data has been incorporated from Center Bureau of Health Intelligence (CBHI), Government of India public database domain (http://www. cbhidghs.nic.in) specifically from National Health Profile of India (NHP), 2005-2008 publication. Data in these domains are developed for cause-specific mortality including vital registration systems, nationally representative surveys, surveillance sites or epidemiological studies. The information obtained from the database are on health and related aspects among the states India and union territories (UT), relevant to diarrheal disease are; 1) Demographic indicators: state/UT wise urban and rural population and state/UT wise sex ratio; 2) Socioeconomic indicators: state/UT wise literacy rate, economic indicators, availability of drinking water and availability of toilets; 3)
Health status indicators: diarrheal disease mortality and morbidity; 4) Health infrastructure indicators: state/UT wise number of sub-centers (SC), primary healthcare centers (PHC) and community healthcare centers (CHC), where SCs are considered to be the smallest units in an area and PHCs being the intermediate while eventually ending into CHCs.

Information is also included from the reports collected from the search engines namely Pub Med, Scopus; and data bank web-links for international agencies for example UN Health Agency and WHO to further understand the relationship at the global level. Number of similar design measures are incorporated and compared are geographical location, frequency of surveillance, size of study population, morbidity and mortality from diarrhoea, sex, population demographics and socio-economic constructs, educational programs; water supply and sewage system, availability and access of pertinent healthcare services, and environmental factors.

\subsection{Statistical Analysis}

Data collected for each of the measures and their yearly variables were analyzed employing statistical processors specifically ANOVA, correlation and regression analysis with SPSS 19.0 and MS Office 2007 statistical tools. State-wide evaluation is performed using autonomic linear models to identify the likeliness of prevalence with the co-occurring measures. All the significant measures consequentially from the statistical analyses are included in the construction of predictioner model and derivations are conducted based on the outcomes analyses and information from the relevant studies. Based on the results obtained, graphs were plotted to demonstrate developments in the yearly incident and death reports, trends in the percentage difference of the prevalence with population and sex ratio as well as the significant correlated parameters.

Insignificant and insufficient analysis outcomes have not been included in the results though were reasoned in brief in the discussion. Less than $5 \%$ of the collected information could not be used during the study evaluation since some congruent data points are not available from the surveys and some, due to their outlying effect size.

\section{Results}

\subsection{Diarrheal Morbidity and Mortality: Yearly Relationship with Population and Sex}

Distinctive patterns of diarrheal morbidity are observed in yearly outcome analysis. Almost all of the relationships have been analyzed at the significant level of $\mathrm{p}<$ 
0.01 otherwise their significance level is described along with. Figure 1 illustrates that the yearly patterns of diarrheal deaths and incidents, suggesting gradual growth in their numbers annually with increasing population. Statistical analysis for identifying the associations between the incidents, death and population patterns showed following outcomes.

In 2005 year, both male and female incidents and their deaths are highly correlated. Male incidents and male deaths are correlated with $r=0.848$; while female incidents and female deaths are correlated with, $r=0.730$. There was also very high correlation, $r=0.9995$ between male and female diarrheal incidents as well as between the male deaths and female deaths, $r=0.976$. Projected population for year 2005 was not available therefore comparative evaluation could not be conducted with the diarrheal reports.

In 2006 year, high correlation $r=0.997$ was present between male and female incidents. Male incidents and deaths correlated with $r=0.716$. Female incidents have correlation, $r=0.593$ with female death. The male incidents correlated to total projected male population with $\mathrm{r}$ $=0.505$ and, female incidents correlated at $\mathrm{r}=0.513$ with female projected population of the year.

In the year 2007, male and female diarrheal incidents were highly correlated with $\mathrm{r}=0.997$ same as in year 2006; and male deaths correlated with female deaths at $\mathrm{r}$ $=0.880$. Male incidents and male deaths were correlated at $\mathrm{r}=0.613$. Female incidents correlated with female deaths at $r=0.834$. Female incidents related with total female projected population of 2007 at $r=0.587$ and female deaths as well correlated highly to the total projected population with $\mathrm{r}=0.442$; while correlation between male incidents and total projected 2007 male population was $r=0.599$.

In year 2008, correlation increased between male and female incidents with $r=0.985$; and male deaths has correlation with female deaths at $r=0.913$. Diarrheal incidents of males correlated with male deaths at $r=$ 0.542 . Total projected male population of yr. 2008 correlated with male deaths at $r=0.536$ though no significant levels were not observed. Female incidents correlated with female deaths at $r=0.650$ and total female projected population at $r=0.578$. Female deaths correlated with projected female population at $\mathrm{r}=0.557$ by significance level of $\mathrm{p}<0.05$.

Even though the numbers of incidents have increased due to population growth; the rate of prevalence in comparison with the population growth has reduced as shown in Figure 2. A further analysis of percentage growth reveals detailed diarrheal patterns comparing with the projected annual population. Yearly rate of percentage growth in the diarrheal prevalence has gradually declined specially in the male population.

There was only a minor growth of $0.36 \%$ in the incident rates; and reduced mortality of $-28.08 \%$ ) during 2007-2008 year comparing with approximately $1 / 2$ fold growth rate in previous yearly reports. Female data also shows substantial effects with gradual decrease both in mortality and morbidity due to diarrhoea in the 2006-

\section{Yearly Diarrheal Incident and Death Reports in India}

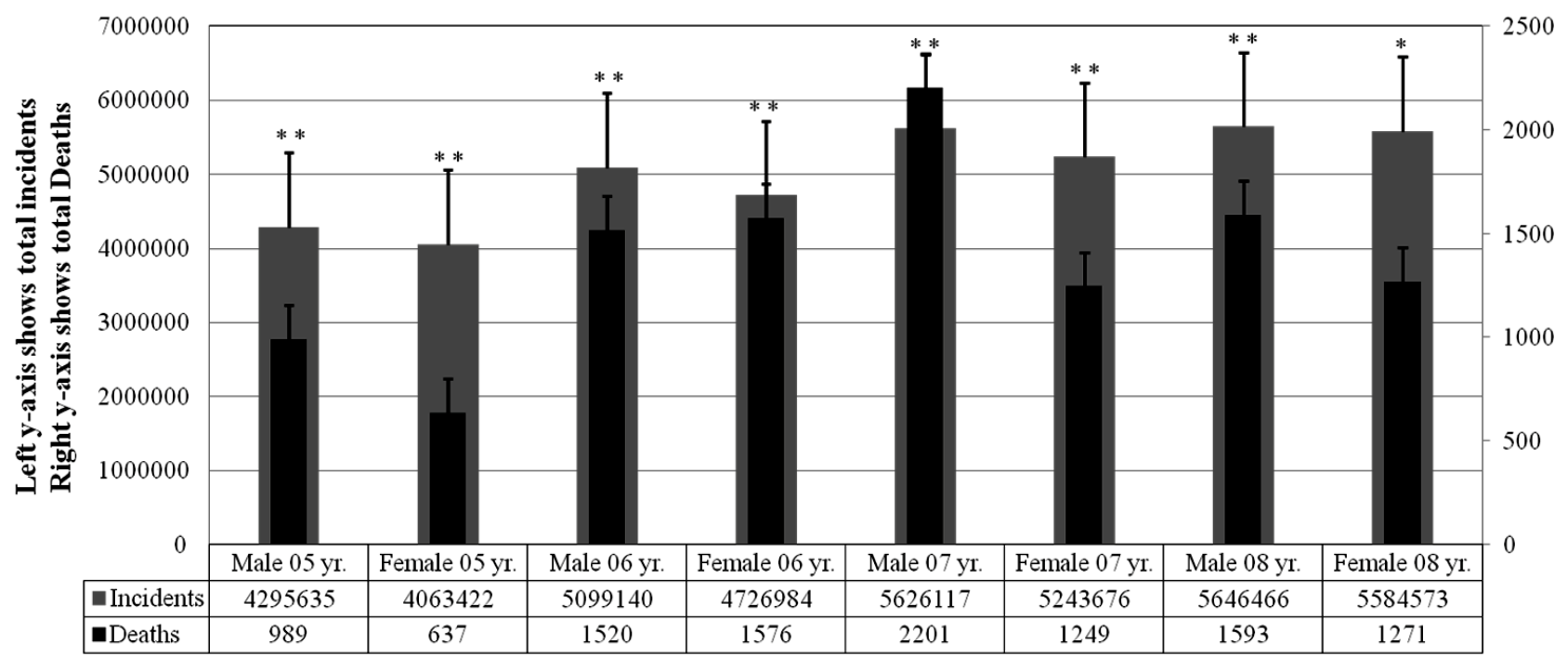

Figure 1. Yearly diarrheal morbidity reports among males and females in India during the period 2005-2008 (Presentation illustrates distribution of sex-based diarrheal incidents and deaths. Both incident and death numbers have increased from 2005year to 2008 year. Very high association is observed between the incidents and deaths annually with significant levels shown as $* * \mathbf{p}<0.01$ and $* \mathbf{p}<0.05)$. 


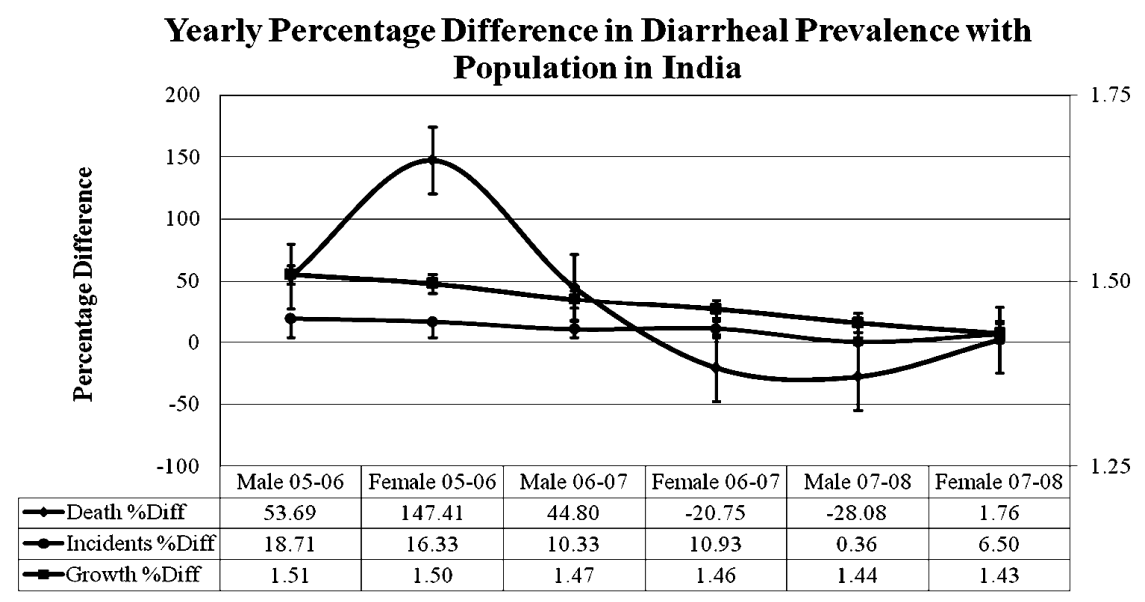

Figure 2. Yearly illustration of percentage difference in the diarrheal incidents and deaths in comparison with the percentage differences in the population growth (Abrupt increase in female incident reports can be observed during 2005-2008 period with gradual lowered and sustained prevalence in following annual data. Males show gradual annual decreases in prevalence from 2005-2006 intervals. Significantly high correlations are evident among the incidents and deaths with the population growth rate annually at $\mathbf{p}<\mathbf{0 . 0 1}$, except $2007-2008 \mathrm{yr}$. female growth rate. Growth rate is plotted on right-hand $y$-axis).

\section{8 investigations.}

Directing appropriate healthcare services by the local, state and central governments gradually towards the proximity of the populations helped lowering the percentage prevalence from $147.41 \%$ growth rates in 2005-2006 to $-20.75 \%$ in females in 2006-2007 year interval, thereby maintaining it thereafter. Linear trend equations also show negative slopes determining further decline in the percentage of diarrhoea prevalence comparing with the population growth.

\subsection{Association with Availability of Healthcare Centers}

Latest information for the healthcare centers has been reported in year 2006 after the launch of 2002 national health care policy of India. The specialty healthcare services for infectious diseases have increased in primary and community centers progressively, thereby helping in restricting the diarrhoea prevalence rates (which demonstrated partial implementations of 2002 Indian national health policy recommendations). Figure 3 provides latest reports on the availability of healthcare centers in India. SCs grew by slow rates of $0.20 \%$ and $0.54 \%$ annually during 2006-2007 and 2007-2008; within the same period, CHCs showed increases of $3.95 \%$ and $5.87 \%$ respectively. Number of PHCs actually reduced with $-1.35 \%$ in $2006-$ 2007 , with slight increase of $2.50 \%$ in $2007-2008$. Further reports do not demonstrate any significant increase in the number of centers.

Although there has not been drastic increase in the healthcare center numbers but the slight inclination seems to manage and regulate the conditions better with time. Yearly relationship analysis from 2006-2008 was con-

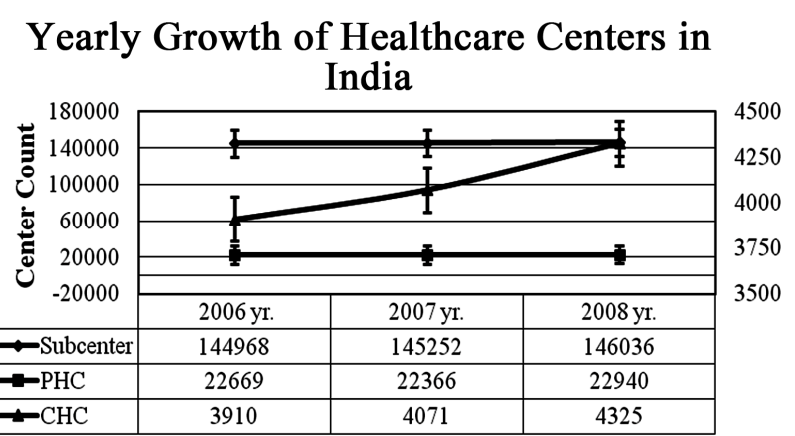

Figure 3. Describes yearly availability of healthcare centers in rural and urban regions in India (All the three kinds of centers have been observing growth in their numbers mostly in the year 2008. PHC numbers actually reduced in numbers in year 2007. CHCs are plotted on the secondary y-axis [right-hand side]).

ducted; including 2005 year to understand the baseline effects in forthcoming years.

Year 2006 incidents of male and female diarrhoea suggests high correlation with PHCs, SCs and CHCs. Numbers of incidents is highly associated with SCs, $r=0.509$ in males and 0.499 in females, that with CHCs, it is $r=$ 0.576 in males and $r=0.556$ in females. Male and female incidents are correlated at $r=0.379$ and $r=0.366$ with significance level of $\mathrm{p}<0.05$ for PHC. Sex-based difference is observed in diarrhoea associated death reports with the centers. Male death data is associated only with SCs, $r$ $=0.348$ and CHCs, $r=0.441$ though female death data could only be related with CHCs, $r=0.352$ at significance level of $p<0.05$. PHCs could not be correlated with either male or female death reports within significant limits.

Year 2007 incidents among males and females also showed very high correlation with availability of PHCs, 
sub centers and CHCs. The correlations for male and female incidents with PHCs were $r=0.474$ and $r=0.442$ respectively with significance level of $\mathrm{p}<0.01$ and $\mathrm{p}<$ 0.05 . SCs were correlated males and females at $r=0.602$ and $r=0.574$ with significance level of $p<0.01$ and $p<$ 0.05 respectively. CHCs were correlated with males $r=$ 0.609 and females $r=0.600$ at the significance level of $p$ $<0.01$. Death reports associated with the healthcare centers less dynamics, male death data only is found to be associated with $\mathrm{CHCs}, \mathrm{r}=0.344$; and female death data was associated with SCs, $r=0.386$ and with CHCs, $r=$ 0.497 within significant levels.

In the year of 2008, similar pattern seems to be repeated with strong correlation of diarrheal incidents among male and female with SCs, PHCs, and CHCs. PHC correlation with male and female incidents were $r=0.429$ and $r=$ 0.442 with significance level of $\mathrm{p}<0.05$. Correlation with SCs were $r=0.589$ and $r=0.594$, while CHCs were correlated with $r=0.537$ and $r=0.553$ with significance level of $\mathrm{p}<0.01$ for male and female population respectively. Male death data could not be associated with any kinds of health centers though female death data is found to be related to SCs, $r=0.377$ and with CHCs, $r=0.452$ within significant limits.

\subsection{Association with Location}

Sex and location has been identified as major measures, which show significant associations with morbidity data (Figure 4). Based on the data evaluation, estimated mapping of the disease pattern are developed, which provide important insights of the disease occurrence.

Based on the linear model paradigm, male and female populations have higher likeliness to have more incidents in the prevalence patterns in some regions chiefly West Bengal, Arunachal Pradesh, and Andhra Pradesh. These regions also have traditionally reported higher mortality rates. High association of male and female morbidity and mortality in previous years also is evident in the graphical interpretations of the predictioner models.

\subsection{Association with Literacy, Water-Supply and Poverty}

The analysis of recent data on the diarrheal incidents and deaths from year 2005-2008 however could not conclude in establishing reverse relationship with literacy, poverty and water supply as contributing factors for restricting disease prevalence as found in western countries [3]. Conducting statistical analysis for the available data only provided positive trends of reverse relationships with literacy, poverty and water supplies. The lack of expected higher significance in the results could be attributed to, insufficient reporting and documentation of data due to lack of awareness, education and economical construct.

\section{Discussion}

Diarrheal diseases are one of the major causes of enormous morbidity, mortality globally; especially in the developing country like India. This, in turn, requires a detailed assessment of the leading and closely related causes of disease. From the previous studies conducted worldwide, it is well known that certain factors such as poor sanitation, education, poverty, health infrastructure and socio-economic conditions play a major role in one or other way to the infectious disease prevalence [16-20]. In the present study we have taken into account some of these major demographic, socioeconomic and health infrastructure indicators in the states and UTs of India to develop an understanding of the prevalence pattern of the disease in the Indian subcontinent. Variables of many measures collectively could identify prototype of diarrheal disease and prevalence characteristics. Statistical analysis suggests high correlation of disease morbidity and mortality with both males and females in the data collected from yrs 2005-2008 suggesting that diarrhoea equally affects both sexes. The disease prevalence were highly associated with the presence of the health care infrastructure such as SCs, PHCs and CHCs suggesting that presence of the health care system not only affects the treatment of the disease but also controls further disease prevalence. Even though both the numbers of incidents and deaths increased along with population growth (Figure 1); the annual growth of prevalence rate has reduced with the simultaneous increase in the association with the health centers suggesting involvements and participation of all the three levels of centers. High correlations of diarrhoea prevalence with population and healthcare services suggest specialized healthcare services can be very efficient in reducing the diarrheal diseases. Stronger association of PHCs in subsequent years may be one of the reasons in reducing the prevalence and distributing the load of the SCs and CHCs. Previous studies in the area also suggests important role of health care infrastructure development in the disease management $[21,22]$.

Community based independent studies performed in the rural and urban area of Nepal and Mali suggests improvement in the diarrheal disease management with the intervention of healthcare volunteers [23,24]. High Diarrheal incident reported in males during the period of 2005-2008 compared to females could be due to less reporting from women having lack of social awareness and education in the area.

In our study of diarrheal prevalence analysis and mapping in the states and UTs of Indian continent there were certain trends of high prevalence in few states which could be useful to strategize the management of the disease in future. We found that trend of diarrheal prevalence was 

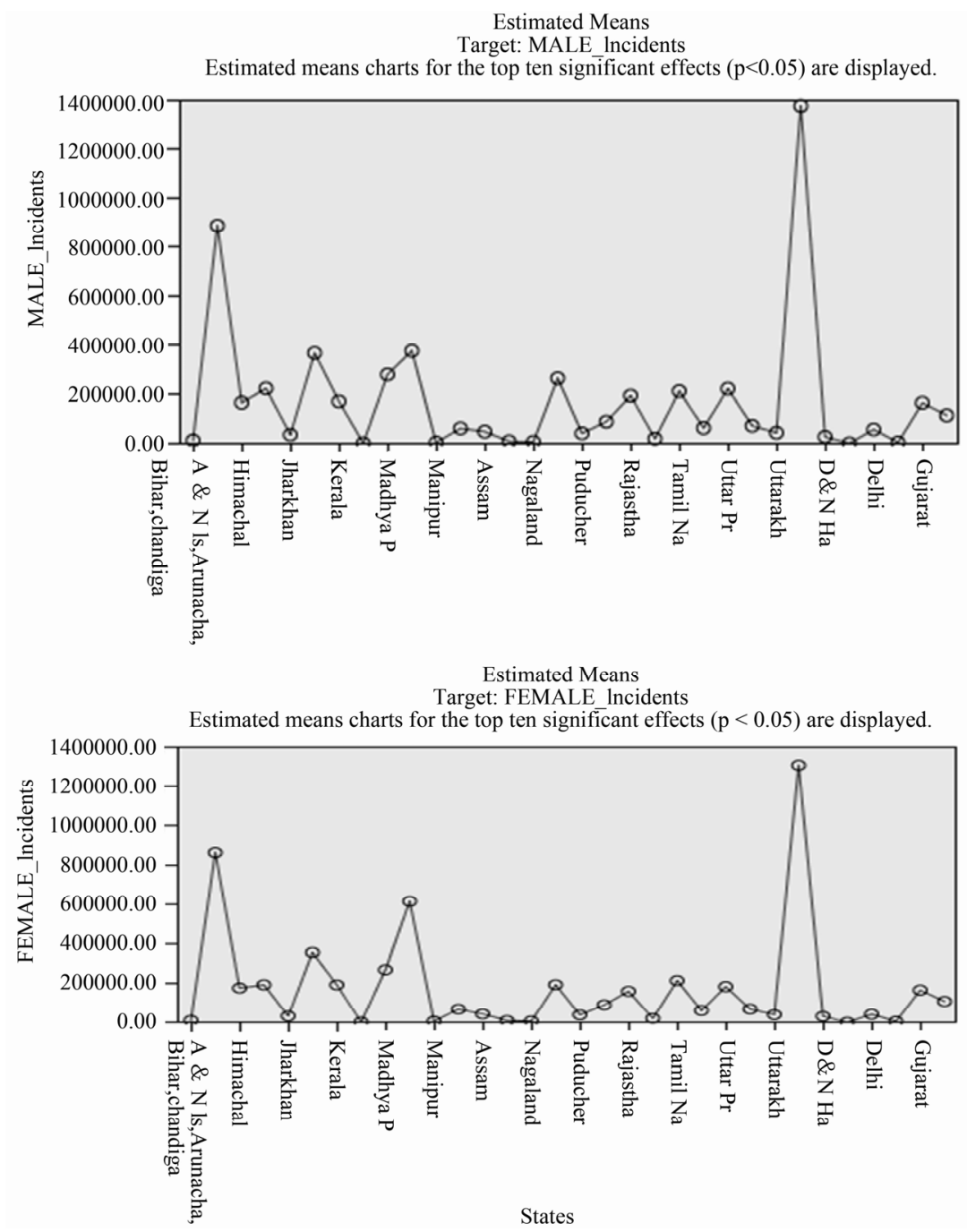

Figure 4. Presentation is derived using autonomic linear model to map the estimated means at significant levels of the incidents due to diarrhea among males and females as a predictor model for identifying prevalence in forthcoming years based on the available data of interactive measures from year 2005-2008 (Statewide morbidity varies with male and female populations).

high in densely populated states like West Bengal, Andhra Pradesh and Aruanchal Pradesh for both males and females. It has already been established in the literature that high infection rate is more likely to occur in densely populated areas due to poor sanitation conditions and close contact between individuals [25].

Availability of clean drinking water, literacy however showed only positive trends of inverse associations; and poverty as positive trends with direct association to the diarrhoea prevalence in our statistical analysis, which can be explained as regional influences helping in identification of state-wide relationships and therefore lacked power in group statistical analysis. Inadequate documentation or underreporting in various regions affected by diarrheal prevalence cannot be ruled out as reasons, which are commonly found in the regions and further planning for accurate documentations and survey structures can reveal local dynamics.

Some more measures can be monitored for inclusion in the governmental surveys, namely, local precursors of outbreaks, trans-generational characteristics of epidemiology and healthcare services management capacity and involvement to further bolster the preventive and therapeutic management of diarrhoea. Genetic predispositions and pathogen virulence variation are also additional areas of study that may supplement information of the prevalence patterns. Such recommendations can be used to include in forthcoming national healthcare policy development. Study is required for further improvement in reliability by incorporating yearly data consistently and evaluating accuracy of data. Evaluation of present prevalence condition in other regions with the model can 
broaden the scope of the surveillance system with demographic validity.

\section{Acknowledgements}

The authors acknowledge International Institute of Health Promotion, Washington DC for project sponsorship and funding; and regional and international agencies namely, WHO and Central Bureau of Health Intelligence (CBHI), Government of India; for the distribution and circulation of healthcare and epidemiological data.

\section{REFERENCES}

[1] World Health Organization, "The World Health Report," WHO, Geneva, 2000.

[2] World Health Organization, "Diarrhoeal Disease Control Programme," Clinical Management of Acute Diarrhea, WHO, Geneva, 1979.

[3] M. J. G. Farthing, "Diarrhea: A Significant Worldwide Problem," International Journal of Antimicrobial Agents, Vol. 14, No. 1, 2000, pp. 65-69. doi:10.1016/S0924-8579(99)00149-1

[4] A. C. Moore, B. L. Herwaldt, G. F. Craun, R. L. Calderon, A. K. Highsmith and D. D. Juranek, "Surveillance for Waterborne Disease Outbreaks—United States, 1991-1992," CDC Surveillance Summaries 42(No. SS-5), MMWR, Atlanta, 1993, pp. 1-22.

[5] A. C. Moore, B. L. Herwaldt, G. F. Craun, R. L. Calderon, A. K. Highsmith and D. D. Juranek, "Surveillance for Waterborne-Disease Outbreaks—-United States, 1993- 1994," CDC Surveillance Summaries 45(No. SS-1), MMWR, Atlanta, 1996, 1996, pp. 1-33.

[6] D. A. Levy, M. S. Bens, G. F. Craun, R. L. Calderon and B. L. Herwaldt, "Surveillance for Water-Borne Disease Outbreaks—United States, 1995-1996," CDC Surveillance Summaries 47(No. SS-5), MMWR, Atlanta, 1998, pp. 134.

[7] D. A. Levy, M. S. Bens, G. F. Craun, R. L. Calderon and B. L. Herwaldt, "Surveillance for Waterborne Disease Outbreaks-United States, 1997-1998," CDC Surveillance Summaries 49 (No. SS-4), MMWR, Atlanta, 2000, pp. 134.

[8] S. H. Lee, D. A. Levy, G. F. Craun, M. J. Beach and R. L. Calderon, "Surveillance for Waterborne Disease Outbreaks -United States, 1999-2000," CDC Surveillance Summaries 51 (No. SS-8), MMWR, 2002, pp. 1-47.

[9] B. G. Blackburn, G. F. Craun, J. S. Yode, V. Hill, R. L. Calderon, N. Chen, S. H. Lee, D. A. Levy and M. J. Beach, "Surveillance for Waterborne-Disease Outbreaks Associated with Drinking Water-United States, 2001-2002," CDC Surveillance Summaries 53 (SS08), MMWR, Atlanta, 2004, pp. 23-45.

[10] J. D. Snyder and M. H. Merson, "The Magnitude of the Global Problem of Acute Diarrheal Disease: A Review of Active Surveillance Data," Bulletin of the World Health Organization, Vol. 60, No. 4, 1982, pp. 605-613.
[11] C. Bern, J. Martines, I. de Zoysa and R. I. Glass, "The Magnitude of the Global Problem of Diarrheal Disease: A Ten-Year Update,” Bulletin of the World Health Organization, Vol. 70, No. 6, 1992, pp. 705-714.

[12] J. E. Tate, S. Chitambar, D. H. Esposito, R. Sarkar, et al., "Disease and Economic Burden of Rotavirus Diarrhea in India,” Vaccine, Vol. 27, Suppl. 5, 2009, pp. F18-24. doi:10.1016/j.vaccine.2009.08.098

[13] A. K. Mukherjee, P. Chowdhury, M. K. Bhattacharya, et al., "Hospital-Based Surveillance of Enteric Parasites in Kolkata,” BMC Research Notes, Vol. 2, 2009, p. 110. doi:10.1186/1756-0500-2-110

[14] S. Gopal, R. Sarkar, K. Banda, J. Govindarajan, et ail., "Study of Water Supply \& Sanitation practices in India Using Geographic Information Systems: Some Design \& Other considerations in a Village Setting,” Indian Journal of Medical Research, Vol. 129, No. 3, 2009, pp. 233-241.

[15] G. B. Nair, T. Ramamurthy, M. K. Bhattacharya, et al., "Emerging Trends in the Etiology of Enteric Pathogens as Evidenced from an Active Surveillance of Hospitalized Diarrheal Patients in Kolkata, India,” Gut Pathogens, Vol. 2, No. 1, 2010, p. 1-4. doi:10.1186/1757-4749-2-4

[16] World Health Organization, "Program for Control of Diarrheal Diseases: Ninth Program Report 1992-1993,” WHO, Geneva, 1994.

[17] World Health Organization, "Water and Sanitation: Fact Sheet 112,” WHO, Geneva, 1996.

[18] P. R. Hunter, "Drinking Water and Diarrheal Disease Due to Escherichia coli," Journal of Water and Health, Vol. 1, No. 2, 2003, pp. 65-72.

[19] S. Nundy, R. H. Gilman, L. Xiao, et al., "Wealth and Its Associations with Enteric Parasitic Infections in a LowIncome Community in Peru: Use of Principal Component Analysis," American Journal of Tropical Medicine and Hygiene, Vol. 84, No. 1, 2011, pp. 38-42. doi:10.4269/ajtmh.2011.10-0442

[20] N. Sastry and S. Burgard, "Changes in Diarrheal Disease and Treatment among Brazilian Children from 1986 to 1996," Population Research and Policy Review, Vol. 30, No. 1, 2011, pp. 81-100. doi:10.1007/s11113-010-9179-9

[21] National Control of Diarrheal Diseases Project, "Impact of the National Control of Diarrheal Diseases Project on Infant and Child Mortality in Dakahlia, Egypt," Lancet, Vol. 2, No. 8603, 1988, pp. 145-148.

[22] L. Rosero-Bixby, "Socioeconomic Development, Health Interventions and Mortality Decline in Costa Rica," Scandinavian Journal of Social Medicine, Vol. 46, Suppl. 46, 1991, pp. 33-42.

[23] L. M. Franco, F. P. Diop, C. R. Burgert, et al., "Effects of Mutual Health Organizations on Use of Priority HealthCare Services in Urban and Rural Mali: A Case-Control Study," Bulletin of the World Health Organization, Vol. 86, No. 11, 2008, pp. 830-838. doi:10.2471/BLT.08.051045

[24] M. Ghimire, Y. V. Pradhan and M. K. Maskey, "Community-Based Interventions for Diarrheal Diseases and Acute Respiratory Infections in Nepal,” Bulletin of the World 
Health Organization, Vol. 88, No. 3, 2010, pp. 216-221. doi:10.2471/BLT.09.065649

[25] R. M. D’Souza, "Housing and Environmental Factors and their Effects on the Health of Children in the Slums of
Karachi, Pakistan,” Journal of Biosocial Science, Vol. 29, No. 3, 1997, pp. 271-281.

doi:10.1017/S002193209700271X 